\title{
Hydrogen Gas Purifiers for Fuel Cells
}

\author{
Marco Succi, Giorgio Macchi, Simona Pirola and Cristian Landoni \\ Saes Getters Spa, Viale Italia 77, Lainate 20020, Milan, Italy
}

\begin{abstract}
Hydrogen is the most common gas used to operate FCs (fuel cells). The performance of proton exchange membrane FCs is sensitive to the hydrogen gas purity. Of particular concern are specific gaseous contaminants such as carbon monoxide, sulphur compounds, and ammonia that are known to drastically reduce the FC efficiency even when present at low concentrations (in the ppb range). To eliminate efficiency losses due to hydrogen purity, dedicated hydrogen gas purifiers are now available specifically for FCs; their adoption protects the FCs and guarantees that consistent gas purity is supplied throughout their lifetime. Different purification technologies have been developed to match the wide variety of applications and to manage various impurities that are dependent on the $\mathrm{H}_{2}$ source. For gas sources where nitrogen is present above the acceptable limit, palladium membrane purifiers can be used to reduce the nitrogen concentration to the desired level. At the same time, the other impurities like carbon monoxide, sulphur compounds, ammonia, hydrocarbons, etc. are also removed. For applications where the main concern is the presence of reactive gases, such as carbon monoxide and sulphur compounds, adsorber purifiers can eliminate these impurities down to the single digit ppb range or better. This technology is suitable to cover a very broad range of flow rates, from a few sccm up to $1,000 \mathrm{~m}^{3} / \mathrm{h}$. The purity performance of both technologies has been proven with state-of-the-art analyzers and will be discussed in the paper.
\end{abstract}

Key words: Fuel cell, purifier, carbon monoxide, impurities, contaminants.

\section{Introduction}

Among the numerous types of FCs (fuel cells), the PEM (proton exchange membrane) is the most sensitive to the presence of gaseous impurities in the hydrogen fuel [1]. The PEM FC is the most widely used type of FC in a number and type of applications, such as stationary applications like back-up power for telecommunications, transport like Materials Handling Vehicles and Light Duty Vehicles [2]. This last application is potentially at the basis of a revolution in the transportation field, where low-efficiency internal combustion engines, emitting a number of dangerous compounds, could be replaced by FCs and electric engines emitting just water vapor [3]. The specifications of the hydrogen used to power the PEM FCs depend on the application: Light Duty Vehicles is the most demanding one, requiring a clean and reliable supply of hydrogen to both preserve the lifetime of the FC stack and to maximize the output power [4].

Corresponding author: Marco Succi, business development manager.
The cleanliness of hydrogen plays a fundamental role in the future adoption of FCs; in fact, the supply of a contaminated $\mathrm{H}_{2}$ batch could irreversibly damage the FC stack or decrease its efficiency [5] and moreover, discourage early adopters of this technology.

Hydrogen purifiers, already developed for other, even more contamination-sensitive applications, such as the electronics industry [6], could be used to eliminate the risk of contamination and increase the reliability of the $\mathrm{H}_{2}$ supply, no matter what the final application is.

This paper will describe two purification technologies that can be used, depending on the specification of the inlet $\mathrm{H}_{2}$, to consistently reach single-digit ppb of all the contaminants that are detrimental for PEM FCs performance.

\section{Scientific Approach}

Palladium membrane purifiers. Pd and Pd alloys, due to both high solubility and diffusivity of $\mathrm{H}_{2}$ in their lattice, are ideal candidates to selectively 
separate hydrogen from gas mixtures. In fact Pd membranes are solid barriers that cannot be crossed by gaseous molecules with the only exception of hydrogen. The mechanism behind this technology is depicted in Fig. 1.

Molecular hydrogen dissociates into atomic hydrogen through chemisorption on the Pd surface, followed by its dissolution in the Pd lattice. Diffusion to the other side of the membrane and recombination into molecular species results into permeation, driven by the difference in (the square root of) $\mathrm{H}_{2}$ partial pressure on both sides of the membrane [7], as described by Sievert's law (the typical appearance of Pd alloy membrane is depicted in Fig. 2, as long as its XRD fingerprint).

Thus only $\mathrm{H}_{2}$ is actually able to permeate through dense Pd-based medium, which therefore acts like a membrane. On the contrary, all other gas molecules are not allowed to pass it, since Pd and Pd alloys behave as an insuperable barrier. Selectivity with respect of $\mathrm{H}_{2}$ is therefore ideal (infinite).

$\mathrm{H}_{2}$ diffusion is driven by the inlet gas pressure and by the $\mathrm{Pd}$ membrane operating temperature (350-400 ${ }^{\circ} \mathrm{C}$ ), with no need of cycling or switching valves during operation. $\mathrm{Pd}$ purifiers have an unlimited lifetime as long as the Pd membrane integrity will be maintained. In terms of footprint, these purifiers are also significantly compact compared to alternative technologies.

In order to keep removing the impurities upstream of the Pd membrane and prevent their build up, a few \%s of the incoming $\mathrm{H}_{2}$ flow, typically $2-4 \%$, is vented along with the impurities [8].

The running cost of this purifier is determined by the power consumption and the loss of $\mathrm{H}_{2}$ from the bleed flow.

Adsorber purifiers. Many transition metals have the ability to react and form stable compounds with gaseous

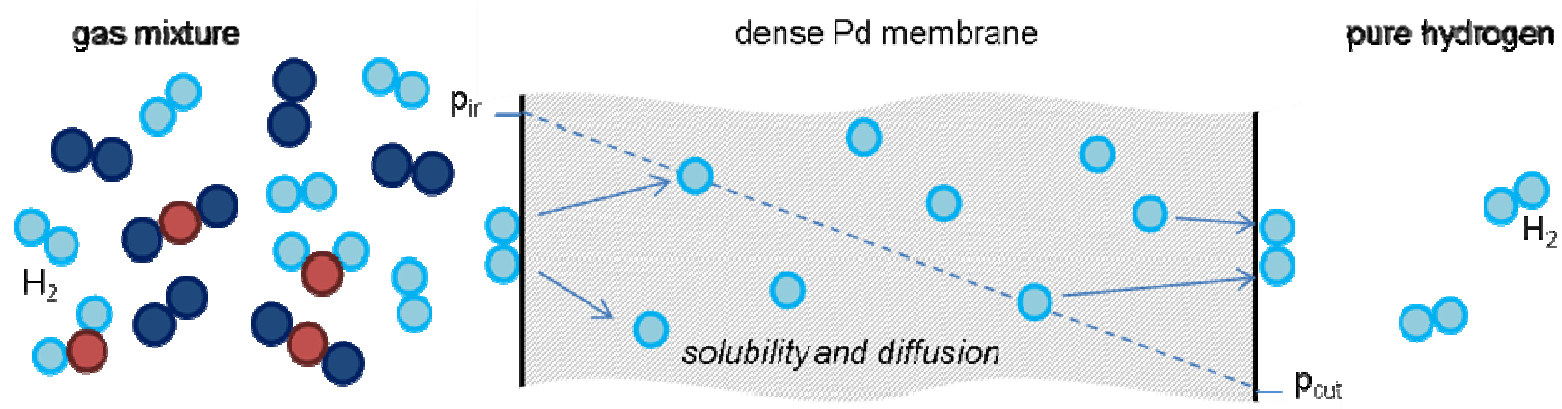

Fig. 1 Pd membrane purifier mechanism.
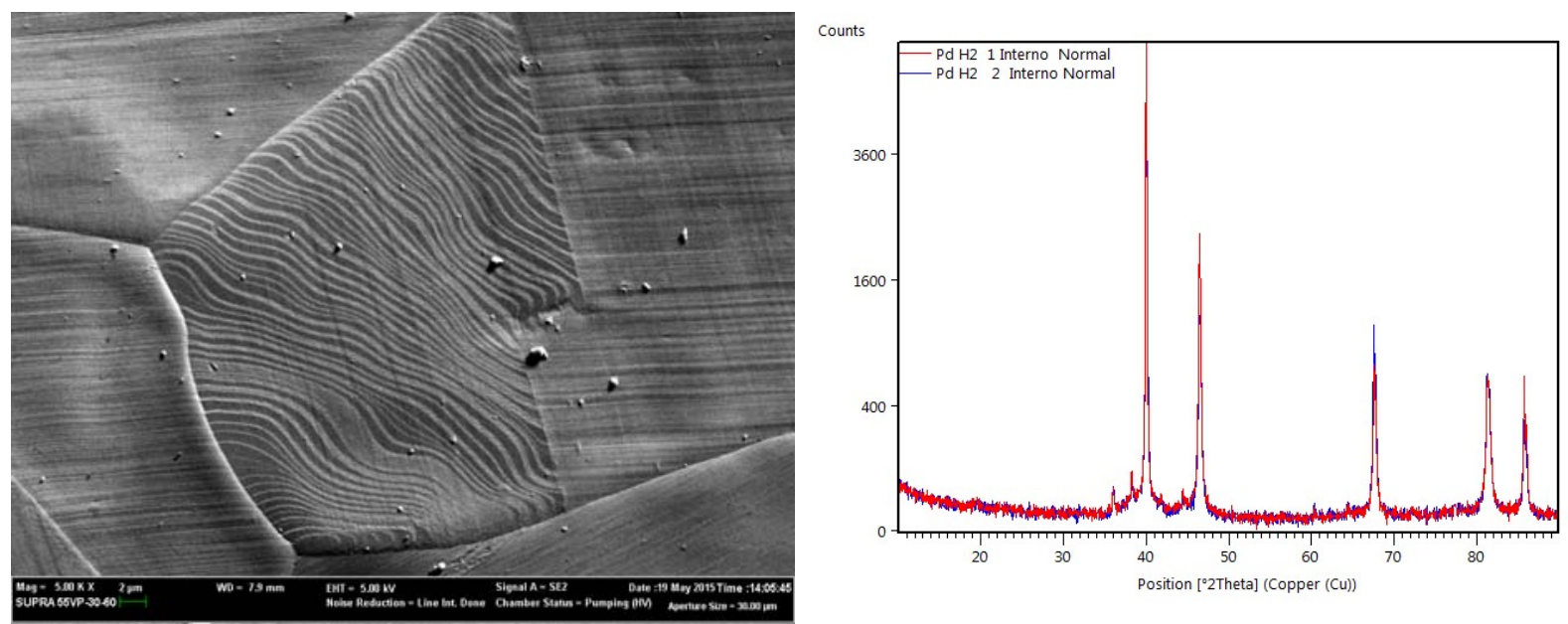

Fig. 2 Typical appearance of Pd membrane as seen by SEM analysis (left) and XRD spectrum of Pd alloy (right). 
molecules on their (activated) surface. Among them, the most effective in $\mathrm{CO}$ and sulphur compounds removal have been identified and used in the form of activated supported media in an adsorber purifier. It is operated at room temperature and, in addition to $\mathrm{CO}$ and sulphur compounds, removes a wide range of reactive impurities such as $\mathrm{O}_{2}, \mathrm{H}_{2} \mathrm{O}, \mathrm{CO}_{2}, \mathrm{NMHC}$ (Non Methane Hydrocarbons), $\mathrm{NH}_{3}$ and $\mathrm{NO}_{\mathrm{x}}$ at ppbv or sub-ppbv levels. It is completely transparent to, and thus not suitable to remove, $\mathrm{N}_{2}, \mathrm{CH}_{4}$ and rare gases.

Once saturated with impurities, the purifier can be regenerated to fully recover the initial capacity and efficiency to sorb impurities. If this technology is used with relatively clean inlet gas, the lifetime estimation of an adsorber purifier could be several years. Lifetime estimation is possible if the average impurity level, the average flow rate, and the duty cycle are known [9].

The cost of ownership of these purifiers is low: in fact they do not require any power to operate or any loss of hydrogen due to venting.

The flow rate managed by these purifiers typically ranges from a few sccm up to $1,000 \mathrm{~m}^{3} / \mathrm{h}$.

\section{Experiments}

The characterization of the tested purifiers was carried out in the laboratory with state-of-the-art analyzers, capable to continuously quantify the concentration of the impurities at the outlet of the purifier to the lowest possible concentration.

Since the capacity of adsorber purifiers is quite high, the tests were carried out with a scaled-down unit, challenged with a high flow rate compared to the real condition. In these accelerated conditions, experimental tests lasted from a few days up to some weeks instead of months or years.

The following analyzers have been used for purifier characterization:

Atmospheric Pressure Ionization Mass Spectrometer [10]. It is a special type of Mass
Spectrometer where the traditional hot filament used to ionize the impurities is replaced by a corona discharge. This technology allows detection of a broad range of impurities and can reach a sensitivity well below $1 \mathrm{ppb}$. Being so sensitive, this technology can only be used when all the impurities in $\mathrm{H}_{2}$ are at least below $1 \mathrm{ppm}$.

GC equipped with a Sulphur Chemiluminescence Detector. It is a detector suitable to analyze sulphur contaminants. To enhance the speed of response of the unit at low concentrations, down to a few ppb, a column with a very weak phase was used. Since it was not able to separate $\mathrm{H}_{2} \mathrm{~S}$ and COS, the two impurities were studied separately.

GC equipped with a Pulsed Discharge Detector. It was used to measure $\mathrm{CO}$ upstream and downstream the Pd purifier. The sensitivity for CO is about $2 \mathrm{ppb}$.

GC equipped with a Flame Ionization Detector. It was also used to measure $\mathrm{CO}$ in $\mathrm{H}_{2}$. This unit was optimized to reach low concentrations with a sensitivity of $2 \mathrm{ppb}$.

To test the performance of a purifier, a synthetic mixture was prepared, starting from high pressure $\mathrm{H}_{2}$ cylinders with $5.0 \mathrm{~N}$ purity and a certified cylinder containing a known amount of the impurity to be tested. By properly tuning a blending system, $\mathrm{H}_{2}$ with the desired impurity concentration, typically in the range of 5-10 ppm, was generated. The hydrogen flow rate was in the range 1-10 slpm.

This mixture was delivered to the purifier under continuous monitoring of the gas quality at its outlet and, in the long term tests, periodically checking the concentration at purifier inlet.

When the purifier reached its end of life, the concentration of the impurity at the outlet started progressively to increase. In this way it is possible to select the best material and process for the removal of the desired impurity (CO, sulphur compounds, etc.) and accurately determine the amount of impurities that can be absorbed by the purifier. 


\section{Results}

Fig. 3 shows the purity achievable by using a $\mathrm{Pd}$ purifier supplied by a $4.8 \mathrm{~N} \mathrm{H}_{2}$ purity, thus containing less than 20 ppm of $\mathrm{N}_{2}, \mathrm{CO}, \mathrm{CO}_{2}, \mathrm{O}_{2}$ and $\mathrm{H}_{2} \mathrm{O}$. None of these impurities pass the Pd membrane and their concentration is reduced down to the detection limit of the analyzer, more than 4 decades lower than the inlet concentration. The flow rate during the test was 10 slpm.

Fig. 4 shows that, even challenging the $\mathrm{Pd}$ membrane purifier with a very high content of CO, up to $140 \mathrm{ppm}$, and a flow rate of $400 \mathrm{sccm}$, the outlet remains at the detection limit of the analyzer, $<2 \mathrm{ppb}$.

Since the presence of sulphur compounds could damage the Pd membrane in the long term, it is preferable to install a purifier for their removal and preserve membrane integrity. Figs. 5 and 6 show the efficiency of the $S$ purifier that reduces the concentration of incoming $\mathrm{H}_{2} \mathrm{~S}$ and COS from almost $12 \mathrm{ppm}$ down to $<2 \mathrm{ppb}$, the sensitivity of the analyzer, at a flow rate of $1.3 \mathrm{slpm}$.

Fig. 7 shows the removal of more than $6 \mathrm{ppm}$ of CO by means of an adsorber purifier including the breakthrough, which is when the CO concentration starts increasing at purifier outlet. The tests were carried out for several days with a $\mathrm{H}_{2}$ flow rate of 4.0 slpm and only the end of the test is shown in the figure.

The adsorber purifier is also quite efficient in removing sulphur compounds as shown in Fig. 8, where no COS was detected at purifier outlet.

\section{Conclusions}

The adoption of FCs is raising more and more interest for their capability to efficiently generate power. Specific applications where they are widely studied and used are: distributed energy generation, back-up power and transportation.

Some types of FCs, for example PEM, are quite sensitive to the presence of contaminants in the $\mathrm{H}_{2}$ fuel.

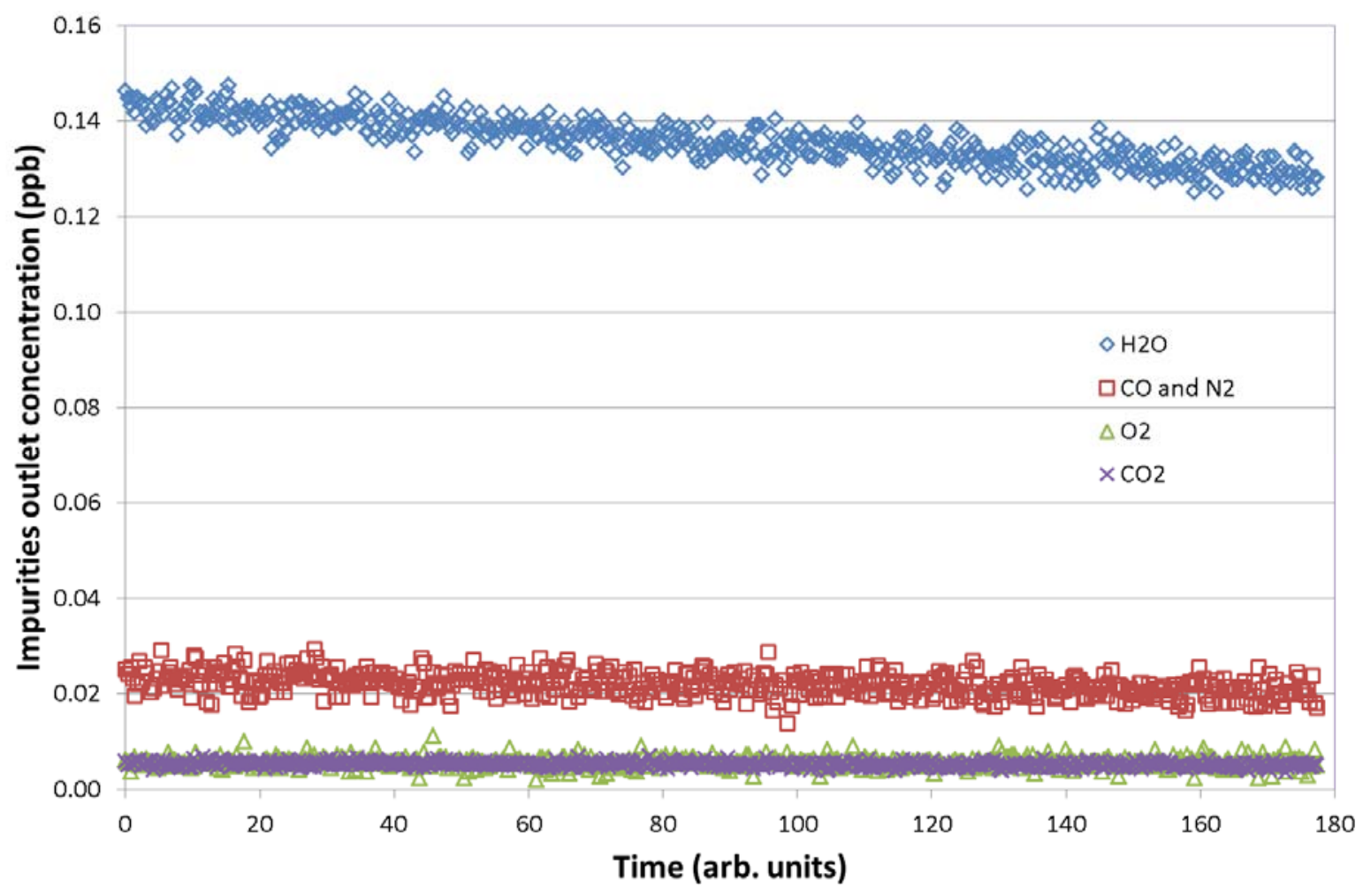

Fig. 3 Pd purifier: typical outlet purity measured by APIMS. 


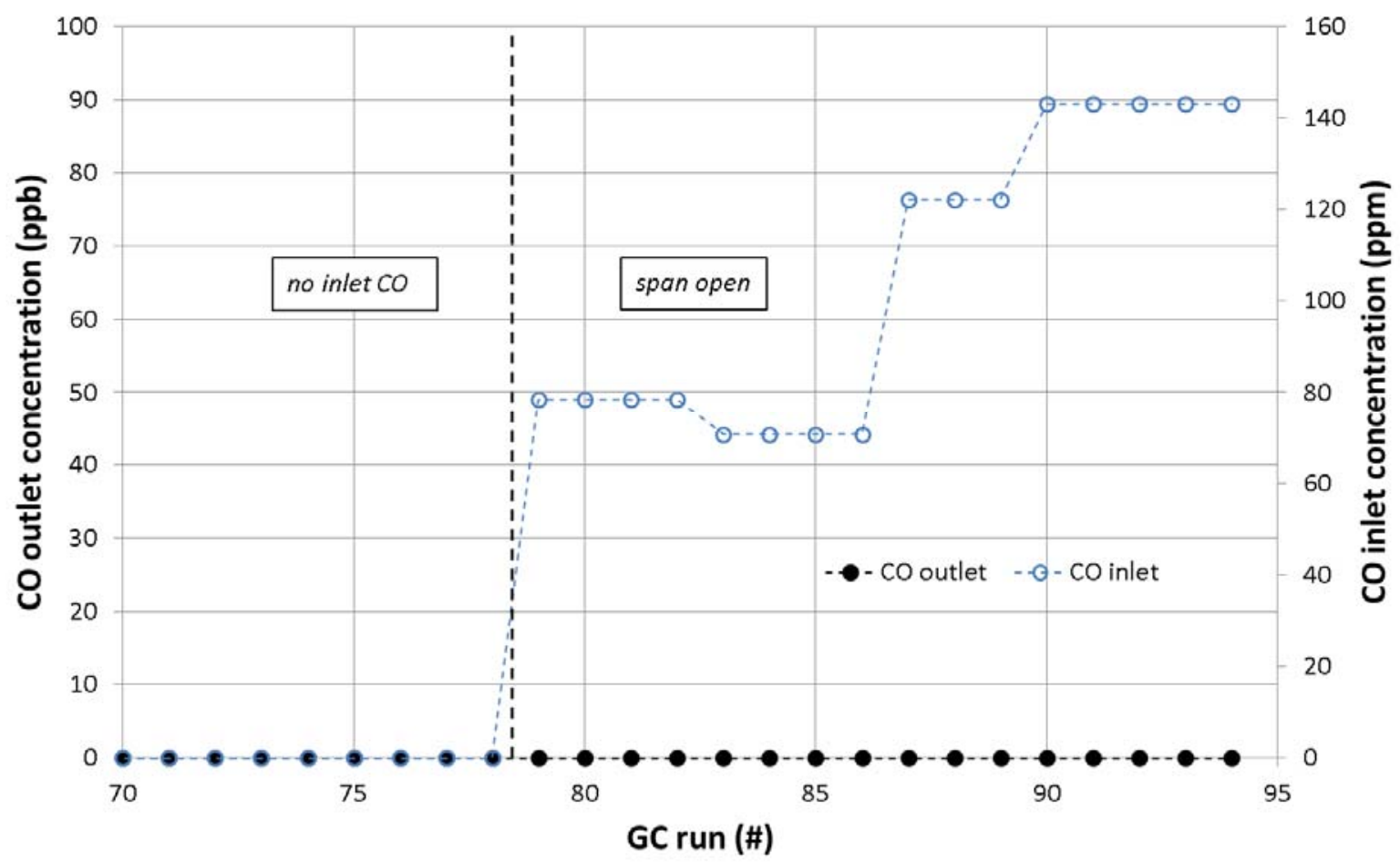

Fig. 4 Pd purifier, CO removal measured by GC-PDD.

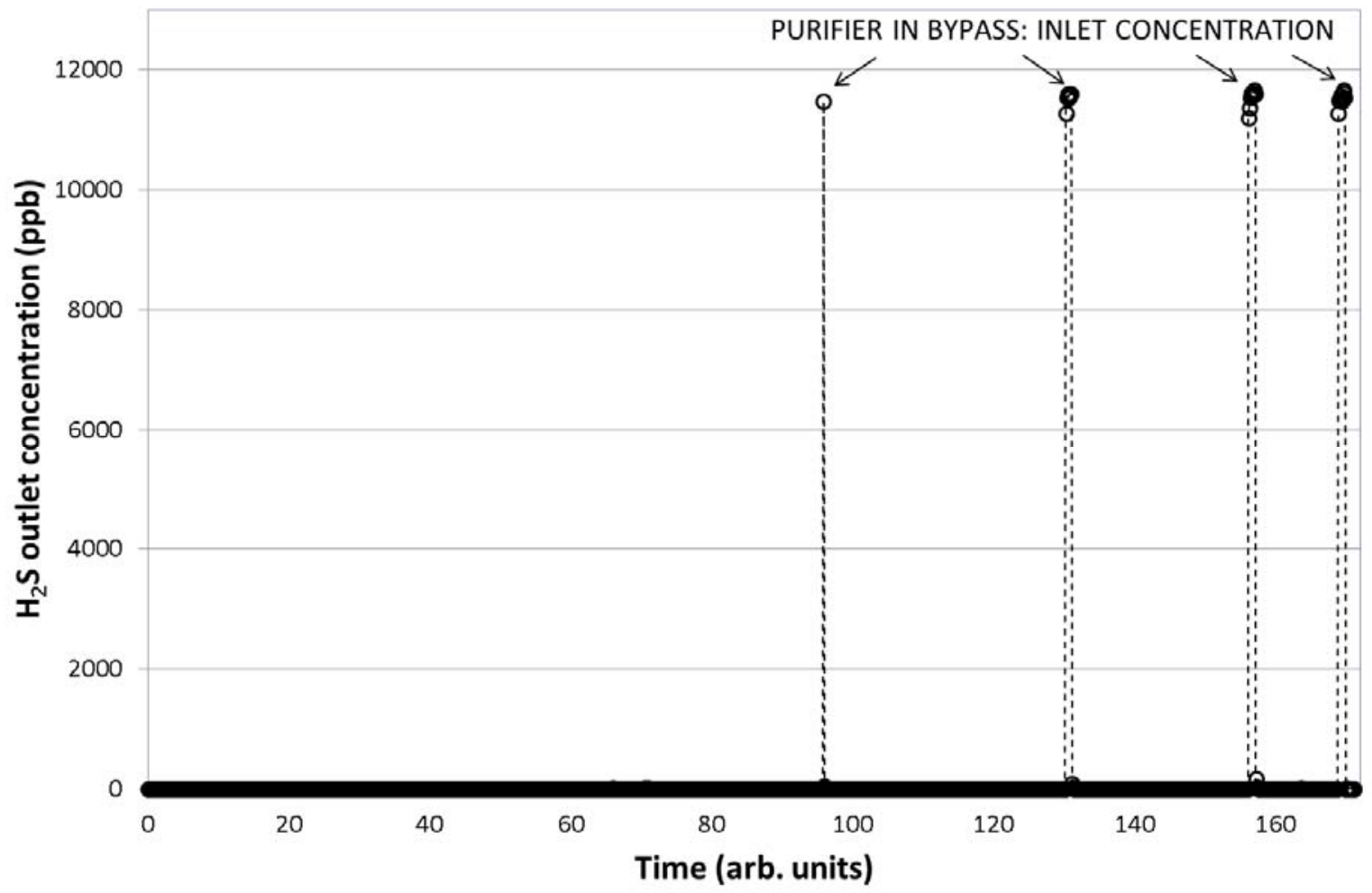

Fig. 5 S purifier, $\mathrm{H}_{2} \mathrm{~S}$ removal measured by GC-SCD. 


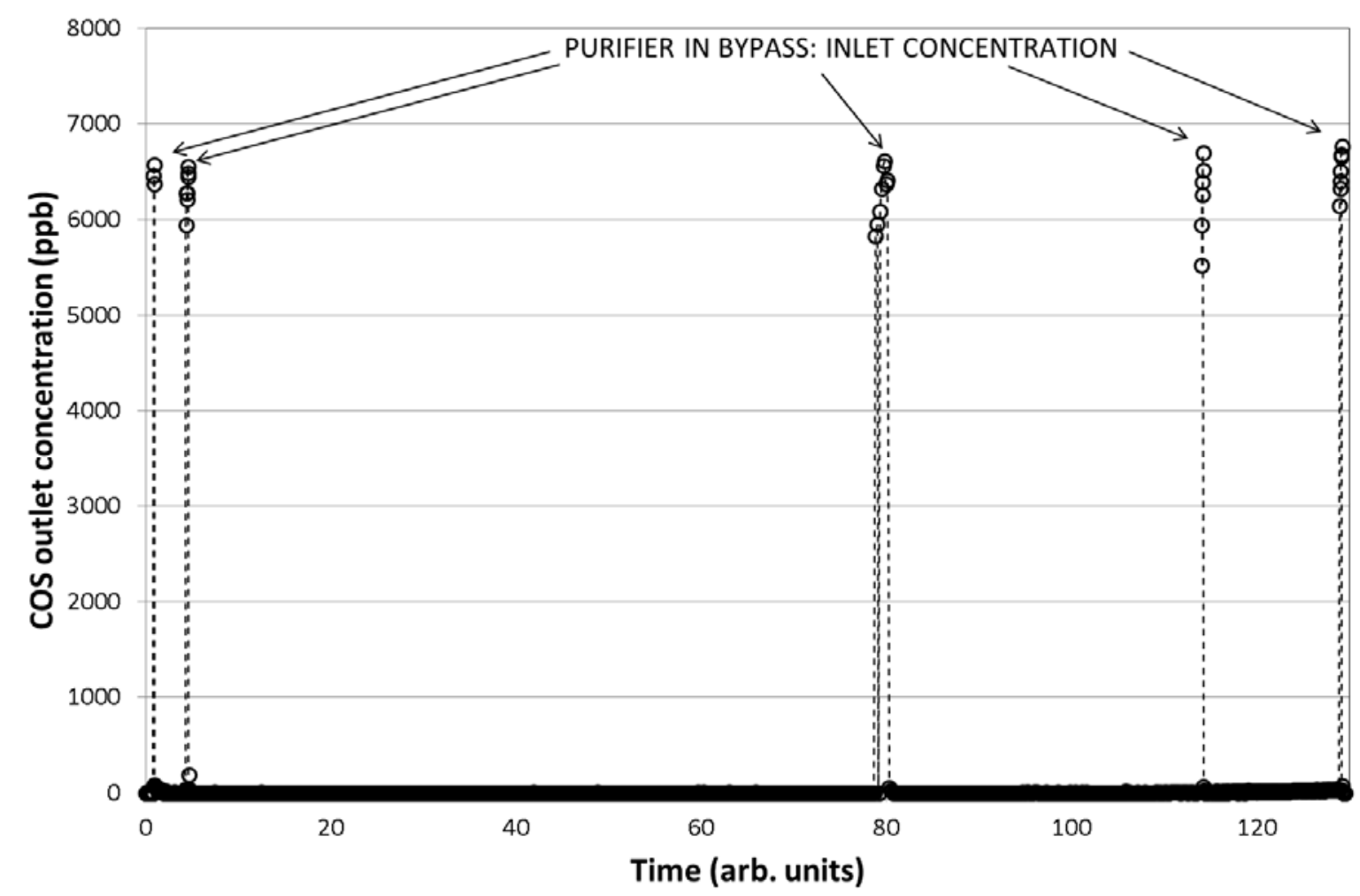

Fig. 6 S purifier, COS removal measured by GC-SCD.

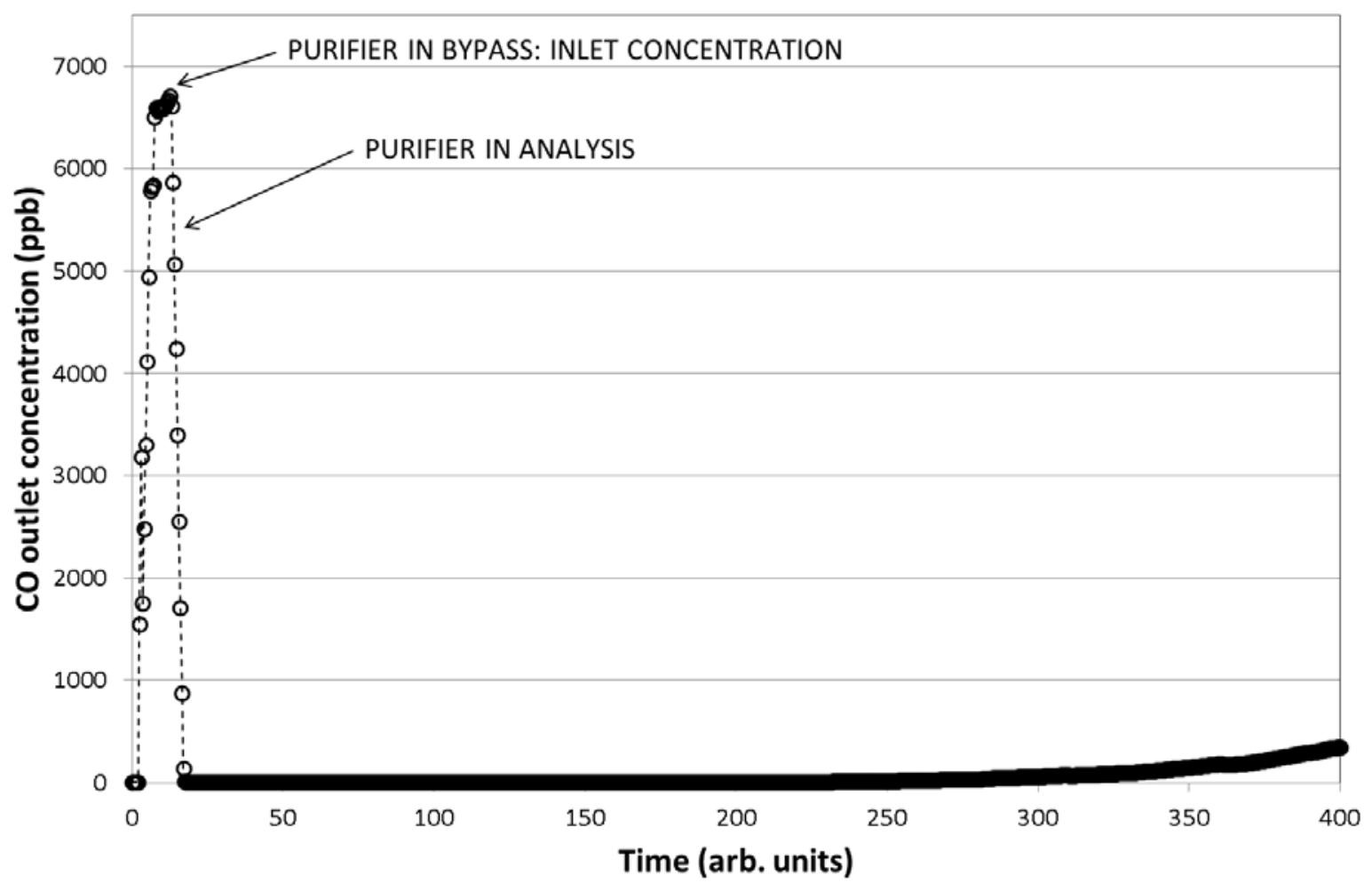

Fig. 7 Adsorber purifier, CO removal measured by GC-FID. 


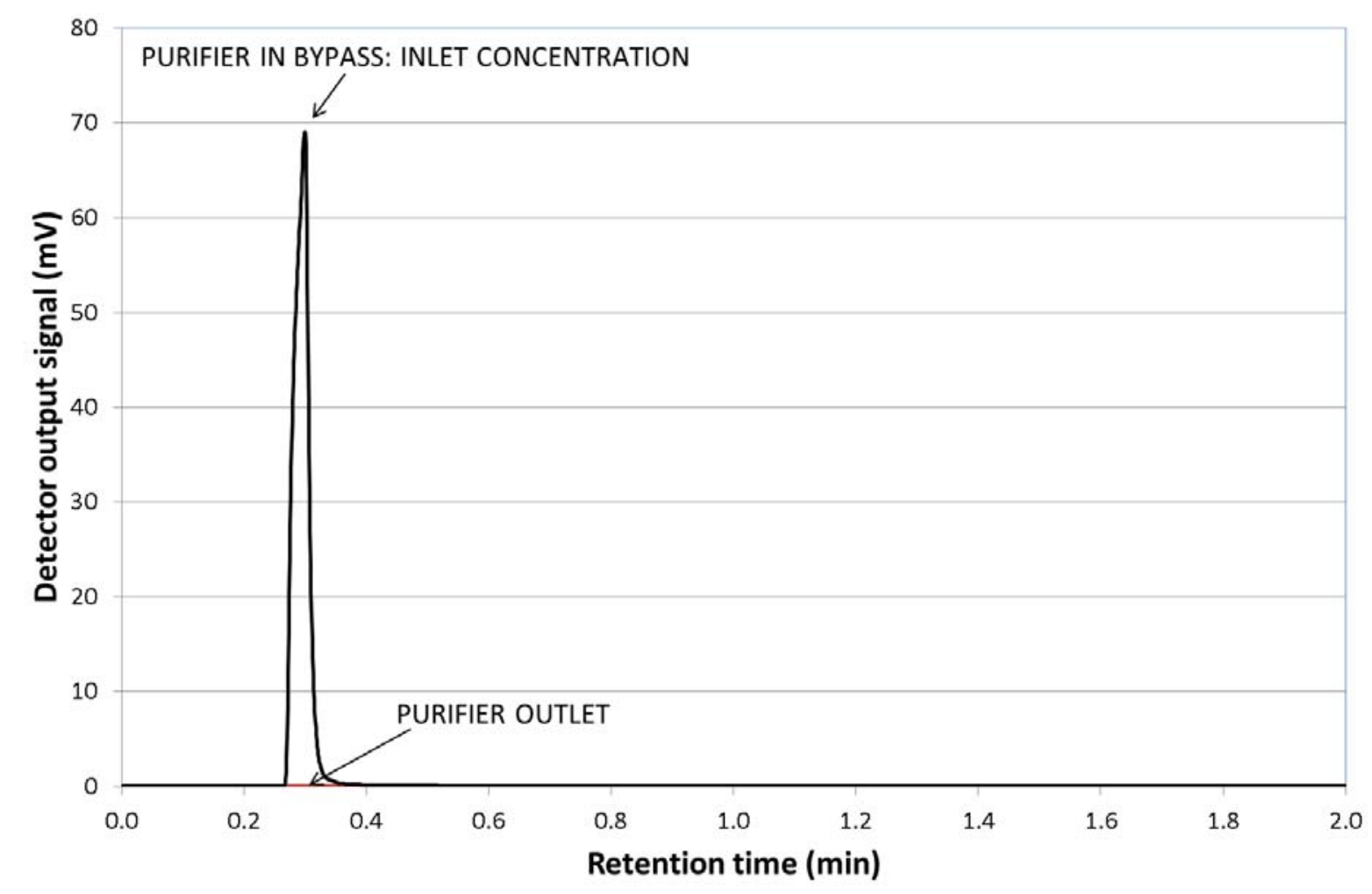

Fig. 8 Adsorber purifier, chromatograms showing S removal measured by GC-SCD.

The use of gas purifiers allows to keep the impurities under control; in particular carbon monoxide and sulphur compounds.

$\mathrm{H}_{2}$ purifiers enhance the reliability of the operation of the FCs eliminating the contamination in $\mathrm{H}_{2}$ fuel among the variables that affect FC operation, by improving its lifetime.

Scientific and technological improvements are continuously addressed to overcome the use of expensive media, such as Pd alloys, by exploiting the potential related to their technology, e.g., by using thinner membrane which results in higher flow rates at low pressure drop.

\section{References}

[1] Rodrigues, A. 1997. "Carbon Monoxide Poisoning of Proton-Exchange Membrane Fuel Cells.” Proceedings of the Honolulu, HI.

[2] The Fuel Cell Industry Review. 2013. Fuel Cell Today.

[3] Ahluwalia, R. 2004. "Fuel Economy of Hydrogen Fuel
Cell Vehicles.” Journal of Power Sources 130 (2004): 192-201.

[4] International Standard ISO 14687-2:2012. Proton Exchange Membrane (PEM) Fuel Cell Applications for Road Vehicles.

[5] Angelo, M. 2008. “The Impacts of Repetitive Carbon Monoxide Poisoning on Performance and Durability of a Proton Exchange Membrane Fuel Cell.” ECS Transactions 16 (2): 669-76.

[6] Ostrander, C. 2001. "The Presence of Impurities in Ultra-High Purity Gas Distribution Systems: Case History Studies." Semiconductor Fabtech (13th edition).

[7] Burkhanov, G. 2011. "Palladium-Based Alloy Membranes for Separation of High Purity Hydrogen from Hydrogen-Containing Gas Mixtures.” Platinum Metals Rev. 55 (1): 3-12.

[8] Leeson, N. 2011. "Developments in the Market for UHP Hydrogen Purifiers.” Semiconductor TODAY Compounds \& Advanced Silicon 6 (4): 116-8.

[9] Briot, O. 2011. "Managing Gas Purity in Epitaxial Growth.” Cryst. Res. Technol. 46 (8): 809-12.

[10] Brieasacher, J. 1991. "Gas Purification and Measurement at the PPT Level.” J. Electrochem. Soc. 138 (12): 3717-23. 